\title{
Experimental characterization of nonlinear interference noise as a process of inter-symbol interference
}

\author{
Ori Golani ${ }^{1}$, , Daniel Elson ${ }^{2}$, Domaniç Lavery $^{2}$, Lidia Galdino ${ }^{2}$, Robert Killey ${ }^{2}$, Polina \\ BAYVEL $^{2}$, AND MARK SHTAIF ${ }^{1}$ \\ ${ }^{1}$ School of Electrical Engineering, Tel Aviv University, Tel Aviv, Israel 69978 \\ ${ }^{2}$ Optical Networks Group, UCL (University College London), Department of Electronic and Electrical Engineering, UCL, Torrington Place, London, UK. \\ ${ }^{*}$ Corresponding author: origolani@post.tau.ac.il
}

We demonstrate a method for experimentally characterizing the second order statistics of nonlinear interference noise (NLIN) as an inter-symbol interference (ISI) process. The method enables to measure the properties of high-order ISI coefficients, which have been largely overlooked in the pass. The ability of measuring these statistics is imperative for designing effective NLIN mitigation schemes. The variance, temporal correlation times, and cross correlations of the various ISI coefficients are evaluated in several system implementations. (C) 2018 Optical Society of America

OCIS codes: (060.4370) Nonlinear optics, fibers; (060.4510) Optical communications.

http://dx.doi.org/10.1364/ao.XX.XXXXXX

\section{INTRODUCTION}

High-speed, wavelength division multiplexed (WDM) systems are often limited by nonlinear interactions between different WDM channels. At each individual WDM receiver, these interactions appear random and are registered as noise. It is customary to refer to this noise as nonlinear interference noise, or NLIN.

In recent years, extensive efforts have been invested into the characterization of NLIN statistics, to explore the extent to which it is really random, and to devise possible methods for its mitigation. An important finding was that the cross-phase-modulation (XPM) component of NLIN (the predominant component in most practical scenarios of high channel count systems operating at a baud rate higher than 10 GBuad in each channel) can be modeled very accurately as an inter-symbol interference (ISI) process with random and time varying ISI coefficients [1-3]. Hence, the characterization of NLIN can be translated into the characterization of the corresponding ISI coefficients. Devising reliable experimental approaches for doing that is imperative for assessing the prospects of NLIN mitigation techniques [4-7].

Almost all existing research to date has considered only the zeroth-order ISI coefficient, whose contribution to NLIN is as phase and polarization rotation noise (PPRN) [8-10]. Yet, in most cases of interest, it is also important to account for higher order ISI terms, whose accumulated effect may exceed the PPRN contribution. One of the obstacles to doing so is the difficulty in evaluating the higher order ISI terms experimentally. When one performs a transmission experiment the contribution of all ISI orders is intertwined, and they must be separated in order to be measured properly. The zeroth-order ISI term produces distinctively distorted, 'kidney-shaped', points in the received constellation, which makes its identification relatively simple. In contrast, the effect of higher order ISI terms is not geometrically obvious, and can be easily confused with additive white noise.

In this Letter, a new method for measuring any-order ISI coefficients and evaluating their correlation properties is presented. We demonstrate the accuracy of the method in several settings by comparing experimental results to computer simulations. Our findings confirm the importance of the high-order ISI contributions to NLIN. Knowledge of the correlation properties of the ISI coefficients can be exploited for designing novel methods for NLIN mitigation. For example, in ref. [6] a detailed model of the correlations of NLIN was used to obtain a maximum likelihood equalizer, which improved the error rate.

\section{THEORY}

Consider a wavelength division multiplexed (WDM) system, in which the channel of interest (COI) propagates in the fiber along with many interfering channels (ICs). At the receiver, the signal is filtered, sampled, and dispersion compensated. It has been shown that under these conditions the nonlinear interference noise is accurately represented by the time-varying ISI model detailed in $[2,11]$. According to this model, the relation between the received and transmitted signal samples is

$$
\underline{s}_{n}=\underline{a}_{n}+i \sum_{l} \mathbf{R}_{l}^{(n)} \underline{a}_{n-l}+\underline{w}_{n},
$$

where the underlined variables represent two-element column vectors and the boldface variables $\mathbf{R}_{l}^{(n)}$ are $2 \times 2$ ISI matrices. The elements of $\underline{s}_{n}$ are the $n$-th samples of the received signal in the two polarizations, the elements of $\underline{a}_{n}$ are the two polarization symbols launched in the $n$-th time-slot, and the elements of $\underline{w}_{n}$ are statistically independent complex circular Gaussian random variables that account for the contribution of amplified spontaneous emission (ASE) noise. The nonlinear interference 
from the ICs is represented by the ISI matrices $\mathbf{R}_{l}^{(n)}$, whose elements are determined by the data transmitted in the ICs [2]. The index $l$ is the ISI-order, and the superscript $n$ accounts for the slow (on the scale of a symbol duration) dependence of the ISI matrix on time. The effect of nonlinear phase-noise, whose measurement in experiments was addressed in [8], is entirely captured by the the trace of the zeroth order ISI matrix $\mathbf{R}_{0}^{(n)}$. Yet, the experimental characterization of all the other ISI terms has not been done previously and is the subject of our current work.

We now describe the procedure that allows extraction of the second-order statistics of the ISI matrices $\mathbf{R}_{l}^{(n)}$ experimentally. For simplicity, the starting point is the operation in the scalar case, i.e. when $\underline{s}_{n}, \underline{a}_{n}, \mathbf{R}_{l}^{(n)}$, and $\underline{w}_{n}$ are treated as scalars. The more general results that correspond to the polarization multiplexed case is introduced subsequently.

The scalar version of Eq. (1) assumes the following form

$$
\begin{aligned}
s_{n} & =a_{n}\left(1+i R_{0}^{(n)}\right)+i \sum_{l \neq 0} R_{l}^{(n)} a_{n-l}+w_{n} \\
& \simeq a_{n} e^{i R_{0}^{(n)}}+i \sum_{l \neq 0} R_{l}^{(n)} a_{n-l}+w_{n},
\end{aligned}
$$

where the transition from the first to the second line is valid within the first-order perturbation analysis that underpins the entire theoretical framework of NLIN analysis [12]. The fact that $R_{0}^{(n)}$ is real-valued [13] indicates that its only effect is to produce phase-noise, whose statistics can be readily extracted from the received constellation diagram [8]. All other ISI terms in Eq. (2) are uncorrelated with $a_{n}$, and therefore, in a constellation diagram, they appear as circular white noise, which is indistinguishable from the contribution of ASE. In what follows we demonstrate a technique that allows measurement of the second-order statistics of $R_{l}^{(n)}$ for all values of $l$. We stress that our goal is to assess the second-order statistics of the ISI terms experimentally, and not to measure their instantaneous values.

In a transmission experiment setup one measures the noisy samples, $s_{n}$. For the purpose of estimating the statistics of $R_{l}^{(n)}$, we assume that the data symbols transmitted on the COI are known. We also assume that the transmitted constellation is symmetric (so that the mean of $a_{n}$ is 0 ) and that it does not include the origin (i.e. all $a_{n} \neq 0$ ). These last two assumptions are not necessary, but they significantly simplify the derivation. Under these conditions, we define the quantity

$$
\tilde{R}_{l}^{(n)}=\frac{s_{n}-a_{n}}{i a_{n-l}}=R_{l}^{(n)}+V_{l}^{(n)},
$$

which serves as an estimate for $R_{l}^{(n)}$. The term $V_{l}^{(n)}$ represents the estimation error, and it is given by

$$
V_{l}^{(n)}=\frac{1}{a_{n-l}}\left[\sum_{l^{\prime} \neq l} R_{l^{\prime}}^{(n)} a_{n-l^{\prime}}-i w_{n}\right] .
$$

As the data symbol $a_{n-l}$ is independent of any of the elements in the brackets, it follows that $V_{l}^{(n)}$ is a white process. The autocorrelation function (ACF) of $\tilde{R}_{l}^{(n)}$ may therefore be expressed as

$$
\begin{aligned}
f_{\tilde{R}_{l}}(\Delta n) & =\lim _{N \rightarrow \infty} \frac{1}{N} \sum_{n=1}^{N} \tilde{R}_{l}^{(n)} \tilde{R}_{l}^{*(n+\Delta n)} \\
& =f_{R_{l}}(\Delta n)+\operatorname{Var}\left[V_{l}^{(n)}\right] \delta(\Delta n),
\end{aligned}
$$

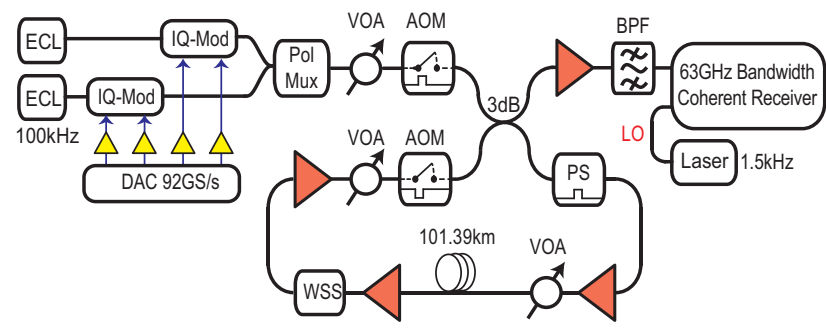

Fig. 1. Experimental setup

where $\delta(\Delta n)$ is the Kronecker delta function. The measured ACF is therefore identical to that of $R_{l}$, with the exception of the point $\Delta n=0$, which correspond to the variance. The cross-correlation function between different ISI elements can also be measured using the same method.

The existence of the delta-correlated term was observed in the past by several researchers who attempted to measure the correlation properties of NLIN-induced phase noise [8], and it hinders the direct measurement of the ISI coefficients' variance. This obstacle is easily circumvented by making use of the fact that the ISI matrices $R_{1}^{(n)}$ are characterized by a moving-average model and therefore their ACF $f_{R_{l}}(\Delta n)$ is smooth. Hence, the value at $\Delta n=0$ may be obtained from the rest of the ACF $(\Delta n>0)$ via interpolation.

The generalization to the dual-polarization case is fairly straightforward. This time we use the estimate

$$
\tilde{\mathbf{R}}_{l}^{(n)}=-i\left(\underline{s}_{n}-\underline{a}_{n}\right) \underline{b}_{n-l}^{T}=\mathbf{R}_{l}^{(n)}+\mathbf{V}_{l}^{(n)}
$$

where $\underline{b}_{n}$ is defined as

$$
\underline{b}_{n}=\left(\begin{array}{c}
a_{x, n}^{-1} \\
a_{y, n}^{-1}
\end{array}\right),
$$

with $a_{x, n}$ and $a_{y, n}$ denoting the two polarization elements of $\underline{a}_{n}$, and the estimation noise matrix $\mathbf{V}_{l}^{(n)}$ is given by

$$
\mathbf{V}_{l}^{(n)}=\sum_{l^{\prime} \neq l} \mathbf{R}_{l^{\prime}}^{(n)} \underline{a}_{n-l^{\prime}} b_{n}^{T}+\mathbf{R}_{l}^{(n)}\left(\begin{array}{cc}
0 & \frac{a_{x, n}}{a_{y, n}} \\
\frac{a_{y, n}}{a_{x, n}} & 0
\end{array}\right)-i \underline{w}_{n} \underline{b}_{n}^{T} .
$$

Similarly to the scalar case, all elements of $\mathbf{V}_{l}^{(n)}$ are white processes, and therefore the ACF of the $i, j$-th element of $\tilde{\mathbf{R}}_{l}^{(n)}$ is given by

$$
\begin{aligned}
f_{\tilde{R}_{l, i j}}(\Delta n) & =\lim _{N \rightarrow \infty} \frac{1}{N} \sum_{n=1}^{N} \tilde{R}_{l, i j}^{(n)} \tilde{R}_{l, i j}^{*(n+\Delta n)} \\
& =f_{R_{l, i j}}(\Delta n)+\operatorname{Var}\left[V_{l, i j}^{(n)}\right] \delta(\Delta n),
\end{aligned}
$$

where $i, j \in[x, y]$.

\section{EXPERIMENTAL SETUP AND RESULTS}

In the experiments the drive signal was generated from sequences of $2^{16}$ random bits mapped to 64 QAM symbols and nonlinearly pre-emphasized using a Volterra filter to compensate for transmitter non-idealities [14]. The symbol rate was 40 GBaud and a root-raised-cosine filter with $0.1 \%$ rolloff was applied. The signals were uploaded to a $92 \mathrm{GSa} / \mathrm{s}$ DAC, linearly amplified and applied to 2 separate IQ modulators that were 


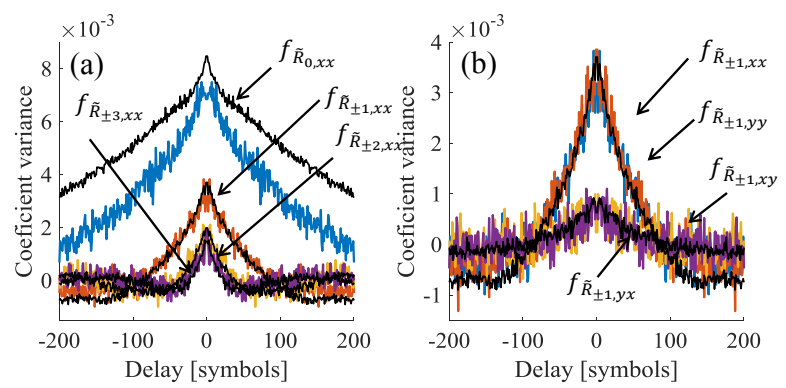

Fig. 2. Measured auto-correlation functions for various ISI coefficients. (a) Shows the ACFs for the diagonal ISI elements, $R_{l, x x}$, for ISI orders $l=0, \pm 1, \pm 2, \pm 3$. (b) shows the ACFs of the four elements of the ISI matrices $\mathbf{R}_{ \pm 1}$. Colored curves correspond to experimental results, while black curves to split-step simulations.In all cases, the delta-correlated term $\operatorname{Var}\left[V_{l, i j}^{(n)}\right] \delta(\Delta n)$ was artificially removed, as to produce a clear figure.

used to independently modulate odd and even WDM channels, which were decorrelated with a 15 ns delay and polarizationmultiplexed. The channel spacing was set to $42.5 \mathrm{GHz}$ and the number of WDM channels was changed between 1 and 15 . The nominal linewidth was $1.1 \mathrm{kHz}$ for the central channel and $100 \mathrm{kHz}$ for the other channels. In all cases, the central channel was used as the COI.

For transmission, a recirculating loop was used as shown in Fig. 1, with a loop-synchronous polarization scrambler and a $101.39 \mathrm{~km}$ span of fibre with a total loss of $16.2 \mathrm{~dB}$. Two EDFAs (5 dB noise figure) separated by a WSS serving as a programmable gain flattening filter were also present inside the loop. The number of times that the signal circulated through the loop was controlled to determine the transmission distance. A single span enabled fine control over transmission distance. The receiver used an ECL with $12 \mathrm{dBm}$ output power and $1.5 \mathrm{kHz}$ nominal linewidth, and balanced photo-diode detectors. The received signals were captured by a real-time digital oscilloscope with an analogue electrical bandwidth of $63 \mathrm{GHz}$ at $160 \mathrm{GSa} / \mathrm{s}$.

The offline digital processing of the traces included IQ delay compensation, chromatic dispersion compensation, matched filtering, polarization demuxing and equalization. A constant modulus algorithm was applied to preconverge 61 taps for a least mean squares radially directed equalizer. The frequency offset between carrier and LO was estimated before a decision directed algorithm was used for carrier phase estimation using 256 taps. Gram-Schmitt orthogonalization was finally performed to remove sub-optimal phase biasing of the transmitter IQ modulators. The experimental results are supplemented by split-step simulations which were performed using the same link parameters specified above. In contrast to the experimental setup, the simulations do not include the effects of ASE, laser phase noise, and the receiver's DSP. Thus, they are used to ascertain that the observed correlations are indeed due to NLIN and not any receiver issues. The number of symbols used in each simulation was $2^{13}$, and 50 independent runs were performed for each configuration, as to obtain smooth results.

Figure 2 shows several examples of the ISI coefficients' autocorrelation functions, for the case of a $20 \times 100 \mathrm{~km}$ link, carrying $7 \mathrm{WDM}$ channels with $2.5 \mathrm{dBm}$ launch power per channel. Figure 2 a shows the ACFs of the $x x$ diagonal elements of seven ISI matrices, corresponding to $l=0, \pm 1, \pm 2, \pm 3$. Due to the presence
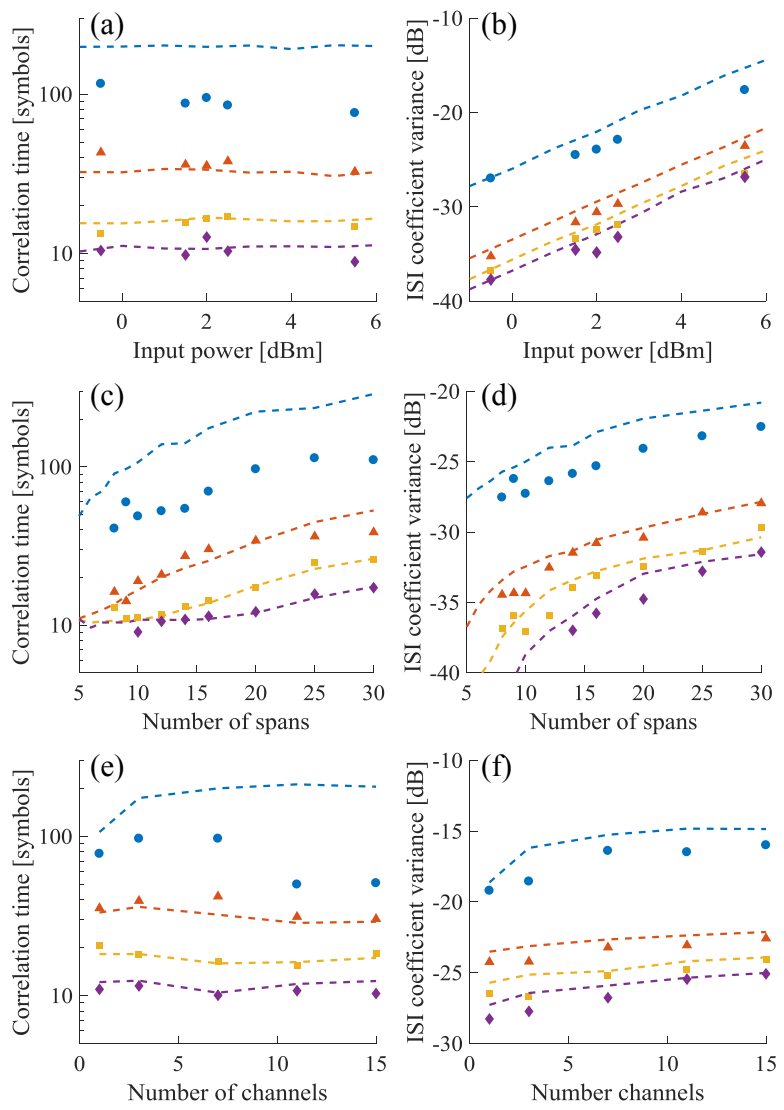

Fig. 3. Correlation time (left column) and variance (right col$\mathrm{umn})$ as a function of power $(\mathrm{a}, \mathrm{b})$, system length $(\mathrm{c}, \mathrm{d})$, and number of WDM channels $(\mathrm{e}, \mathrm{f})$. In all cases the COI is at the center. The figures show $R_{0, x x}$ (blue-dots), $R_{1, x x}$ (red triangles), $R_{2, x x}$ (yellow rectangles), and $R_{3, x x}$ (purple diamonds). Simulations are shown by dashed curves. The number of WDM channels in panels a- $d$ is 7 ( 3 on each side of the COI). The power in $\mathrm{c}-\mathrm{d}$ was $2 \mathrm{dBm}$, and that of $\mathrm{e}-\mathrm{f}$ was $5.5 \mathrm{dBm}$. The number of $100 \mathrm{~km}$ spans in panels a,b,e, and f, was 20 .

of ASE noise, the ACFs of higher order ISI coefficients could not be adequately resolved in the resolution of the figure. Note that elements that correspond to the same $|l|$ (e.g. $R_{1, x x}$ and $R_{-1, x x}$ ) have the same autocorrelation properties, although the values of the coefficients themselves at any given moment are different. It can be seen that all of the coefficients are characterized by correlation times of the order of tens of symbols. Figure $2 b$ shows the four elements of the \pm 1 ISI matrices, $\mathbf{R}_{ \pm 1}$. As can be expected from symmetry, the ACFs of the two diagonal terms are identical, as are the ACFs of the two off-diagonal terms. In addition, the diagonal elements are considerably stronger than the off-diagonal elements, although the ratio between them depends on the transmission parameters (e.g. the variance of the diagonal elements depends on the modulation format, whereas that of the off-diagonal elements does not [2]). In all cases except $l=0$, the agreement between the measured and simulated results is excellent. The discrepancy in the case of $l=0$ is likely due to the laser phase-noise and the phase recovery algorithm that was used in the case of the experimental data. Attempts to include the same algorithm in the simulation failed to produce consistent results.

We estimated the actual ACFs from the noisy measurements 
by fitting the positive side of the measured ACFs $(\Delta n>0)$ to a 5 -th order polynomial function. The variance was estimated to be the fitted function's value at $\Delta n=0$, whereas we define the correlation time as the point where the correlation drops to $e^{-1}$ of its peak. Figure 3 shows the dependence of the ISI coefficients' variance and correlation time on the launch-power, the length of the system, and the number of interfering WDM channels. As expected [2], the variance of the ISI coefficients is proportional to the square of the launch power, but the correlation time remains independent of it. It can be seen that both the variance and correlation time of all coefficients increase with the link's length, as shown in Figs. 3c and 3d. The growth in variance is simply due to the accumulation of larger nonlinearity, whereas the growth in the correlation time is attributed to the larger overall walk-off between the channels. The gradual growth of the variance with the number of WDM channels in Fig. 3f is consistent with the fact that the total NLIN increases when more ICs are added into the system. As expected, the growth of NLIN is seen to be more pronounced in the behavior of $\mathbf{R}_{0}^{(n)}$, which accounts for PPRN. This is caused by the fact that the added WDM channels are spectrally farther from the COI, in which case their nonlinear interaction with it is characterized by a larger fraction of complete collisions [15]. Yet, the weak dependence of the correlation time on the number of channels seen in Fig. 3e, is somewhat surprising in view of the increasingly fast walk-off that characterizes the interaction with each additional IC that is added into the system. Notice that while in cases where $l \neq 0$ the experimental results are generally in good agreement with the simulations, the case of $l=0$ is characterized by various irregularities. Most obvious of which is the non-physical variations of the correlation time of $R_{0, x x}$ with the launch power in Fig. 3a and with the number of WDM channels in Fig. 3e. As noted earlier, this phenomenon is likely due to the effects of laser phase-noise and to the stability of the phase-recovery algorithm, which seems to produce different results at different operating points. Attempts to reproduce this behavior in simulations were unsuccessful.

Lastly, in Fig. 4 we demonstrate the use of the proposed technique for evaluating the cross-correlations between different ISI orders. The correlations between the $l= \pm 1$ ISI orders are considered in Fig. 4a, whereas Fig. $4 \mathrm{~b}$ focuses on the correlation between ISI orders $l=0$ and $l=1$. All curves were obtained only for the $x x$ elements of the involved matrices and normalized to the square root of the product of the corresponding variances, such that the value at zero delay yields Pearson's correlation coefficient. Notice that the cross correlation functions of the real parts of $R_{x x, \pm 1}^{(n)}$ and of the imaginary parts of $R_{x x, 1}^{(n)}$ in Fig. 4 a peak very close to 1 , and -1 , respectively. This indicates that $R_{x x, 1}^{(n)}$ and $R_{x x,-1}^{(n)}$ are almost exactly the complex conjugates of one another. The fact that the peak absolute value of the experimental curves in Fig. 4a is slightly larger than 1 is due to measurement noise. A much weaker correlation characterizes the relation between ISI orders $l=0$ and $l=1$ shown in Fig. $4 \mathrm{~b}$. In general, the strong correlations exist between coefficients corresponding to ISI orders of opposite sign (such as \pm 1 , or \pm 2 etc.). This correlation is strongest for the $l= \pm 1$ coefficients and reduces gradually as $|l|$ increases. The correlation between all other pairs of coefficients is significantly smaller. This interesting behavior arises from the particular structure of the ISI matrices (see Eq. (8) in [12], and Eq. (7) in [13]). The nonlinear interaction coefficients of opposite-sign pulses are very similar, leading to this result.

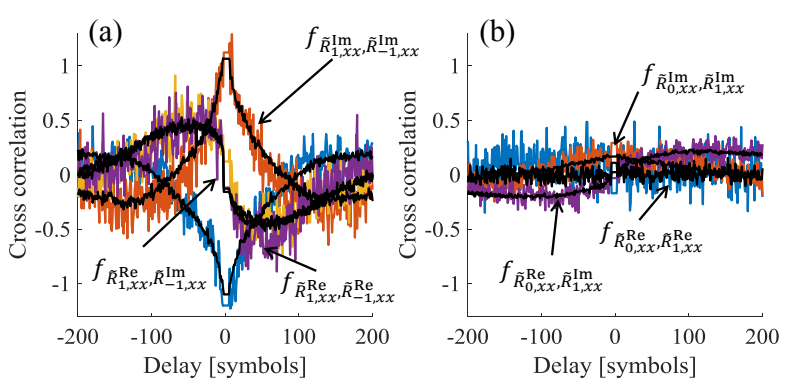

Fig. 4. Normalized cross-correlation functions of the $x x$ elements of the $l_{1,2}= \pm 1$ ISI matrices (a) and the $l_{1}=0$ and $l_{2}=1$ ISI matrices (b). The functions are plotted separately for combinations of the the real and imaginary parts. The coefficients' real and imaginary parts are denoted by $R_{l, x x}^{\mathrm{Re}}$ and $R_{l, x x}^{\mathrm{Im}}$, respectively.

\section{CONCLUSIONS}

We proposed a method of measuring the statistical properties of NLIN, as described by the time-varying ISI model. Estimating the variance of these coefficients is crucial for assessing the potential gains of nonlinearity mitigation by means of equalization, whereas the correlation times are needed to determine the required tracking speed. Furthermore, a such correlation measurements can be used to improve NLIN mitigation, as shown in [6]. It was shown that although the largest contribution to NLIN comes from the zeroth-order ISI (PPRN), the accumulated contribution of higher-order ISI terms is significant. The proposed method allows the experimental evaluation of the autocorrelation functions of the elements of the ISI matrices, as well as the cross-correlations between elements of different ISI orders.

\section{REFERENCES}

1. M. Secondini and E. Forestieri, Photon. Tech. Lett. 24, 2016 (2012).

2. O. Golani, R. Dar, M. Feder, A. Mecozzi, and M. Shtaif, J. Lightwave Tech. 34, 3482 (2016)

3. R. Dar, M. Feder, A. Mecozzi, and M. Shtaif, Opt. Express 22, 14199 (2014).

4. D. Zibar, M. Piels, R. Jones, and C. G. Schäeffer, J. Lightwave Tech. 34, 1442 (2016).

5. M. Yankov, F. Da Ros, E. P. da Silva, T. Fehenberger, L. Barletta, D. Zibar, L. Oxenloewe, M. Galili, and S. Forchhammer, J. Lightwave Tech. 35, 1438 (2017).

6. O. Golani, M. Feder, and M. Shtaif, in OFC, (2017), pp. W2A-46.

7. M. Secondini and E. Forestieri, Photon. Tech. Lett. 26, 2252 (2014).

8. P. Poggiolini and Y. Jiang, J. Lightwave Tech. 35, 458 (2017).

9. T. Fehenberger, M. Mazur, T. A. Eriksson, M. Karlsson, and N. Hanik, in ECOC, (2016).

10. C. Schmidt-Langhorst, R. Elschner, F. Frey, R. Emmerich, and C. Schubert, in ECOC, (2015).

11. O. Golani, M. Feder, A. Mecozzi, and M. Shtaif, in OFC, (2016), p. W31.2.

12. R. Dar, M. Feder, A. Mecozzi, and M. Shtaif, J. Lightwave Tech. 33, 1044 (2015).

13. R. Dar, M. Feder, A. Mecozzi, and M. Shtaif, Opt. Express 21, 25685 (2013).

14. P. W. Berenguer, M. Nölle, L. Molle, T. Raman, A. Napoli, C. Schubert, and J. K. Fischer, J. Lightwave Tech. 34, 1739 (2016).

15. R. Dar, M. Feder, A. Mecozzi, and M. Shtaif, J. Lightwave Tech. 34, 593-607 (2016). 


\section{FULL REFERENCES}

1. M. Secondini and E. Forestieri, "Analytical fiber-optic channel model in the presence of cross-phase modulation," Photonics Technology Letters 24, 2016-2019 (2012).

2. O. Golani, R. Dar, M. Feder, A. Mecozzi, and M. Shtaif, "Modeling the bit-error-rate performance of nonlinear fiber-optic systems," Journal of Lightwave Technology 34, 3482-3489 (2016).

3. R. Dar, M. Feder, A. Mecozzi, and M. Shtaif, "Accumulation of nonlinear interference noise in fiber-optic systems," Opt. Express 22, 1419914211 (2014).

4. D. Zibar, M. Piels, R. Jones, and C. G. Schäeffer, "Machine learning techniques in optical communication," Journal of Lightwave Technology 34, 1442-1452 (2016).

5. M. Yankov, F. Da Ros, E. P. da Silva, T. Fehenberger, L. Barletta, D. Zibar, L. Oxenloewe, M. Galili, and S. Forchhammer, "Nonlinear phase noise compensation in experimental WDM systems with 256QAM," Journal of Lightwave Technology 35, 1438 - 1443 (2017).

6. O. Golani, M. Feder, and M. Shtaif, "Kalman-MLSE equalization of nonlinear noise," in "Optical Fiber Communication Conference," (Optical Society of America, 2017), pp. W2A-46.

7. M. Secondini and E. Forestieri, "On XPM mitigation in WDM fiber-optic systems," Photonics Technology Letters 26, 2252-2255 (2014).

8. P. Poggiolini and Y. Jiang, "Recent advances in the modeling of the impact of nonlinear fiber propagation effects on uncompensated coherent transmission systems," Journal of Lightwave Technology 35, 458-480 (2017).

9. T. Fehenberger, M. Mazur, T. A. Eriksson, M. Karlsson, and N. Hanik, "Experimental analysis of correlations in the nonlinear phase noise in optical fiber systems," in "ECOC 2016; 42nd European Conference on Optical Communication; Proceedings of," .

10. C. Schmidt-Langhorst, R. Elschner, F. Frey, R. Emmerich, and C. Schubert, "Experimental analysis of nonlinear interference noise in heterogeneous flex-grid WDM transmission," in "European Conference on Optical Communication (ECOC), 2015," (IEEE, 2015), pp. 1-3.

11. O. Golani, M. Feder, A. Mecozzi, and M. Shtaif, "Correlations and phase noise in NLIN- modelling and system implications," in "Optical Fiber Communication Conference," (Optical Society of America, 2016), p. W3I.2.

12. R. Dar, M. Feder, A. Mecozzi, and M. Shtaif, "Inter-channel nonlinear interference noise in WDM systems: modeling and mitigation," Journal of Lightwave Technology 33, 1044-1053 (2015).

13. R. Dar, M. Feder, A. Mecozzi, and M. Shtaif, "Properties of nonlinear noise in long, dispersion-uncompensated fiber links," Opt. Express 21, 25685-25699 (2013).

14. P. W. Berenguer, M. Nölle, L. Molle, T. Raman, A. Napoli, C. Schubert, and J. K. Fischer, "Nonlinear digital pre-distortion of transmitter components," Journal of Lightwave Technology 34, 1739-1745 (2016).

15. R. Dar, M. Feder, A. Mecozzi, and M. Shtaif, "Pulse collision picture of inter-channel nonlinear interference in fiber-optic communications," Journal of Lightwave Technology 34, 593-607 (2016). 